\title{
Hanseníase no município de Fortaleza, CE, Brasil: aspectos epidemiológicos e operacionais em menores de 15 anos (1995-2006)
}

\author{
Hansen's Disease in the municipality of Fortaleza, CE, Brazil: epidemiological and operational \\ aspects in children under I5-years-old (1995-2006) \\ La Lepra en el municipio de Fortaleza, CE, Brasil: aspectos epidemiológicos \\ yoperacionales en menores de 15 años (1995-2006)
}

\section{Carlos Henrique Morais de Alencar', Jaqueline Caracas Barbosa', Alberto Novaes Ramos Jr', Maria de lesus Freitas de Alencar", Ricardo José Soares Pontes', Cláudio Gastão JunQueira de Castro'", Jorg Heukelbach'}

'Universidade Federal do Ceará. Faculdade de Medicina, Departamento de Saúde Comunitária. Fortaleza, CE "Secretaria Estadual de Saúde de Rondônia. Programa de Controle de Hanseníase e Tuberculose. Porto Velho, RO

"I'Universidade de São Paulo. Faculdade de Saúde Pública, Departamento de Práticas. São Paulo, SP

\section{RESUMO}

Estudo transversal Que objetiva analisar a ocorrência de casos de hanseníase em menores de 15 anos de idade residentes no Município de Fortaleza e notificados no SINAN. Os indicadores epidemiológicos nesta população mostram hiperendemicidade. Operacionalmente observa-se a manutenção da concentração de atendimento em algumas unidades de referência, apesar de alguns avanços. Diagnóstico tardio, elevado grau de incapacidade no diagnóstico e baixo grau de avaliação de contatos registrados revelam a fragilidade das ações de controle. Ressalta-se a possibilidade de erro diagnóstico frente às características da infecção nesta população. A ocorrência de casos de hanseníase nesta população representa um indicador epidemiológico de grande relevância e sua análise amplia a discussão sobre problemas operacionais na rede de serviços de saúde.

Descritores: Hanseníase; Criança; Epidemiologia descritiva; Prevenção \& controle.

\section{ABSTRACT}

This is a sectional study that aims to analyze the occurrence of Hansen's disease cases in 15 year-old minors in the Municipality of Fortaleza and notified in SINAN. The epidemiological indicators in this population show hiperendemicity. In operational terms is observed the maintenance of the service concentration in some reference units, in spite of some progress. Late diagnosis, a high degree of incapacity in the diagnosis and low degree of evaluation of contacts registered reveal the fragility of the actions of control. This study emphasizes the possibility of error diagnostic front to the characteristics of the infection in this population. The occurrence of cases of leprosy in this population represents an epidemiologic indicator of great importance and its analysis extends the discussion on operational problems in the network of health services.

Descriptors: Leprosy; Child; Epidemiology, descriptive; Prevention \& control.

\section{RESUMEN}

Estudio transversal con el objetivo de analizar las ocurrencias de los casos de lepra en menores de 15 años de edad sitiados en la ciudad de Fortaleza y enterados en el SINAN. Los indicadores epidémicos en ésta población se presentaron hiperendémicos. En la operacinalización, se observa el mantenimiento de la concentración de atención en algunas unidades recomendables, a pesar de algunos avances. Diagnósticos muy tardes, gran grado de incapacidad en el diagnóstico y bajo grado de evaluación de contactos registrados, muestran la fragilidad de las acciones de control. Se resalta así la posibilidad de error de diagnóstico delante de las características de la infección en ésta población. La ocurrencia de casos de lepra en ésta población representa un indicador epidémico de gran importancia y su análisis amplia la discusión sobre los problemas operacionales en la red de servicios públicos.

Descriptores: Lepra; Niño; Epidemiología descriptiva; Prevención \& control.

Financiamento: Edital MCT - CNPQ/MS - SCTIE - DECIT- No 35/2005 
Hanseníase no município de Fortaleza, CE, Brasil: aspectos epidemiológicos e operacionais em menores de 15 anos no período de 1995 a 2006

\section{INTRODUÇÃO}

A hanseníase ainda mantém-se como um processo infeccioso crônico de elevada magnitude em vários países. Apesar disto, de uma forma ampliada, o caráter de doença negligenciada compromete sistematicamente o seu controle, apesar dos avanços alcançados ${ }^{(1,2)}$. Um dos indicadores epidemiológicos mais importantes em termos da sinalização de dinâmica de transmissão recente é a ocorrência de casos em menores de Quinze anos de idade ${ }^{(2,3)}$. Este indicador sinaliza para a existência de fontes humanas ativas de infecção.

Segundo dados da Organização Mundial de Saúde (OMS), em todo o mundo, 254.525 casos novos foram detectados no ano de 2007, no início de 2008. O Brasil contribuiu com 15,4\% (39.125) desses casos, sendo o país com maior número de casos no continente Americano (93,2\% - 39.125 entre 41.978 casos do continente) ${ }^{(1)}$.

Dados nacionais revelam Que no período de 2001 a 2006 o Brasil vinha mantendo uma média anual de 47.400 casos novos detectados em todas as idades, com um parâmetro alto de endemicidade (critério OMS), especialmente nas regiões Norte, Nordeste e Centro-Oeste. A média mais alta dos coeficientes acumulados por macrorregiões variou de 6,6/10.000 habitantes na Região Norte, 5,8/10.000 habitantes na Região Centro-Oeste e 3,4/10.000 habitantes na Região Nordeste. Para os menores de Quinze anos de idade, o país manteve nesse período uma média de 4.000 casos novos detectados $(8,4 \%)$. Ressalta-se que um total de 387 municípios foi responsável por 70\% (16.613 / 23.853) dos casos novos de hanseníase nesta faixa etária ${ }^{(3)}$.

No ano de 2007 foi notificado um total de 39.27 I casos novos de hanseníase no país, a maior parte na região Nordeste (16.335, $41,6 \%$ dos casos do país). O Estado do Ceará foi responsável por 2.510 casos $(6,4 \%$ dos casos do país e $15,4 \%$ dos casos da região Nordeste). O país mantém um coeficiente médio de detecção em menores de 15 anos de idade de 0,53 casos novos por 10.000 habitantes (número absoluto 2.980 casos novos), considerado como muito alto. Nos últimos cinco anos, ressalta-se que a média de casos novos detectados no Brasil nessa população foi de $4.000 \operatorname{casos}^{(4)}$.

Esses dados são significativos, tanto que o Programa Nacional de Controle da Hanseníase (PNCH) nos últimos três anos vem sinalizando este indicador como prioritário para o monitoramento da doença. A região nordeste foi responsável por $47,0 \%$ de todos os casos em menores de 15 anos de idade no país em 2007. O Estado do Ceará isoladamente foi responsável por $5,5 \%$ de todos os casos em menores de 15 anos de idade do país, o $6^{\circ}$ lugar entre todas as unidades da Federação em termos de número absoluto ${ }^{(4)}$.

Uma das Questões importantes, mas pouco abordada, não apenas no Brasil, mas em outros países endêmicos, é o padrão epidemiológico desigual de ocorrência da hanseníase (e de outras endemias) nos territórios ${ }^{(5,6)}$. Alguns estudos realizados na região Nordeste do Brasil, mais especificamente no Ceará, vêm contribuindo para uma maior compreensão desse aspecto da epidemiologia da doença. Municípios com maior desigualdade social apresentaram os maiores coeficientes de detecção e de prevalência de hanseníase, reforçando Que indicadores sócio-econômicos e ambientais também se mostram importantes preditores da hanseníase ${ }^{(7-10)}$. Portanto, estudos relacionados aos aspectos epidemiológicos e operacionais da hanseníase, uma doença expressa em territórios e populações negligenciados, principalmente nas periferias dos grandes centros urbanos são totalmente justificados. De fato, há uma necessidade premente de delineamento de áreas vulneráveis socialmente ou de maior risco para a hanseníase no sentido de se estruturarem de forma mais clara as ações de controle $\mathrm{e}^{(5,11)}$.

Frente a este cenário e considerando-se a especificidade do Mycobacterium leprae pelo acometimento de nervos periféricos bem como o potencial de geração de alterações motoras e sensoriais potenciais, impactos significativos do ponto de vista físico, social e econômico ampliam a carga deste processo infeccioso ${ }^{(12)}$. O presente estudo tem como objetivo analisar a situação epidemiológica da hanseníase em menores de 15 anos no Município de Fortaleza, no período de 1995 a 2007.

\section{METODOLOGIA}

O presente estudo caracteriza-se como inter-relacional transversal. Foram utilizados dados secundários a partir do Sistema de Informação de Agravos de Notificação (SINAN) específico para casos de hanseníase na população na faixa-etária de 0 a 14 anos, residente no Município de Fortaleza, Ceará, no período de 1995 a 2007. A base de dados foi obtida junto à Célula de Vigilância Epidemiológica da Secretaria de Saúde do Município, salvaguardandose a confidencialidade dos dados de identificação de cada caso.

O Município de Fortaleza, capital do Estado do Ceará ( 1725 ), localizado no litoral norte da região Nordeste. Possui 313,14 km² de área (0,2 I\% do estado), com densidade demográfica de 7.85 I,27 habitantes $/ \mathrm{km}^{2}$ em 2007. É atualmente a Quarta maior capital do país em termos populacionais, estimando-se sua população residente total no ano de $2007 \mathrm{em} 2.458 .545$ pessoas. A taxa de crescimento anual estimada da população em termos percentuais nos períodos de 1980-1991, 1991 a 2000 e 2000-2007 é de, respectivamente, $2,0 \%, 2,8 \%$ e $2,2 \%$.

O processamento e a análise dos dados foram feitos a partir dos softwares TabWin (DATASUS) e Excel (Microsoft $\left.{ }^{\circledR}\right)$. Para a avaliação dos indicadores epidemiológicos e operacionais nesta população foram considerados os parâmetros da OMS preconizados pelo Ministério da Saúde. Para o coeficiente de detecção em menores de 15 anos: hiperendêmico Quando acima de 1,0 por 10 mil habitantes; muito alto de 0,5 a 0,99 por 10 mil habitantes; alto de 0,25 a 0,49 por 10 mil habitantes; médio de 0,05 a 0,24 por 10 mil habitantes; e baixo Quando inferior a 0,05 por 10 mil habitantes (MS, 2007; MS 2002)

Os dados demográficos relativos à distribuição anual da população no Município de Fortaleza foram obtidos a partir do Instituto Brasileiro de Geografia e Estatística (IBGE).

\section{RESULTADOS E DISCUSSÃO}

Os dados do estudo refletem uma grande fragilidade de desenvolvimento e integração das ações na rede de atenção primária. O caráter de doença negligenciada da hanseníase é fato no Município de Fortaleza. Sua magnitude e transcendência são indiscutíveis, principalmente em áreas periféricas da capital. Reconhece-se Que a ocorrência da hanseníase é influenciada pelo perfil genético do hospedeiro mas, principalmente, por características ambientais como condição socioeconômica / nutricional, vacinação BCG e taxas de exposição a $M$. leprae ou a outras micobactérias ${ }^{(13,14)}$. 
Observa-se uma elevada proporção de detecção de casos em crianças ao longo dos últimos 13 anos (Gráfico I). Considerando-se a possibilidade de subnotificação de $\operatorname{casos}^{(15)}$, inclusive nesta população específica, aumenta-se ainda mais a dimensão da doença no município.

Por outro lado, o correto diagnóstico da hanseníase é de fundamental importância sob todos os aspectos relacionados à epidemiologia, ao manejo dos casos e à prevenção das incapacidades $^{(16)}$. No caso dos menores de Quinze anos de idade, uma das justificativas possíveis para o número excessivo de casos notificados em crianças é o diagnóstico incorreto, por erros na abordagem diferencial com outras doenças dermatológicas ou neurológicas ${ }^{(4)}$.

Observa-se uma detecção crescente de casos, mantendo uma tendência crescente ao longo dos últimos 13 anos, com uma média de 95 casos/ano. Em Fortaleza, o coeficiente de detecção em menores de 15 anos no período de 1995 a 2007 foi de 1,44 (variando de 0,93 a 2,23) por dez mil habitantes. Mesmo com uma Queda nos dois últimos anos, provavelmente relacionada a Questões operacionais pela intensidade, ainda assim os valores são muito altos e traduzem uma tendência média geral de crescimento.

Pode ser observada uma peQuena variação no número de casos

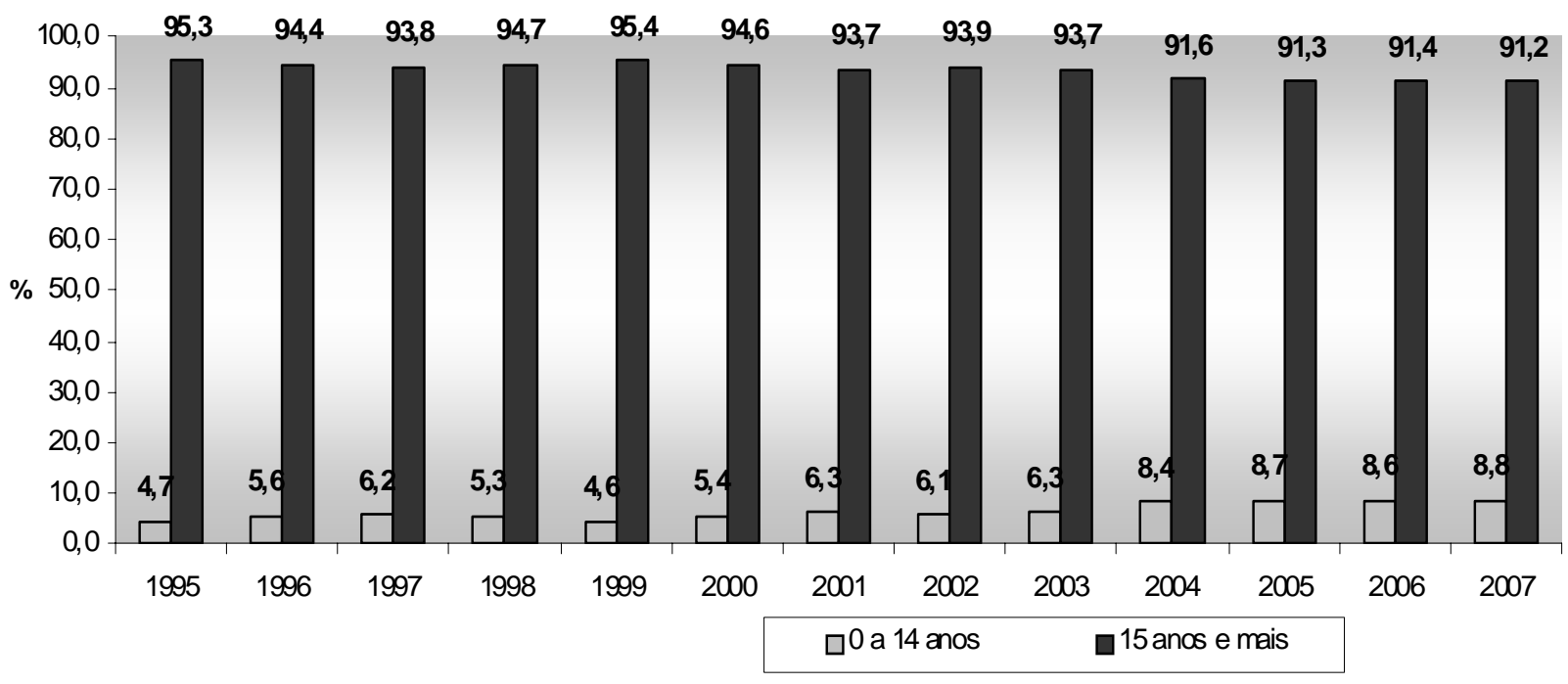

Fonte: SINANNET - SMS/Fortaleza (Dados Preliminares até 30/07/2008)

Gráfico 1. Proporção de casos novos de hanseníase por faixa etária no município de Fortaleza nos anos de 1995 a 2007.

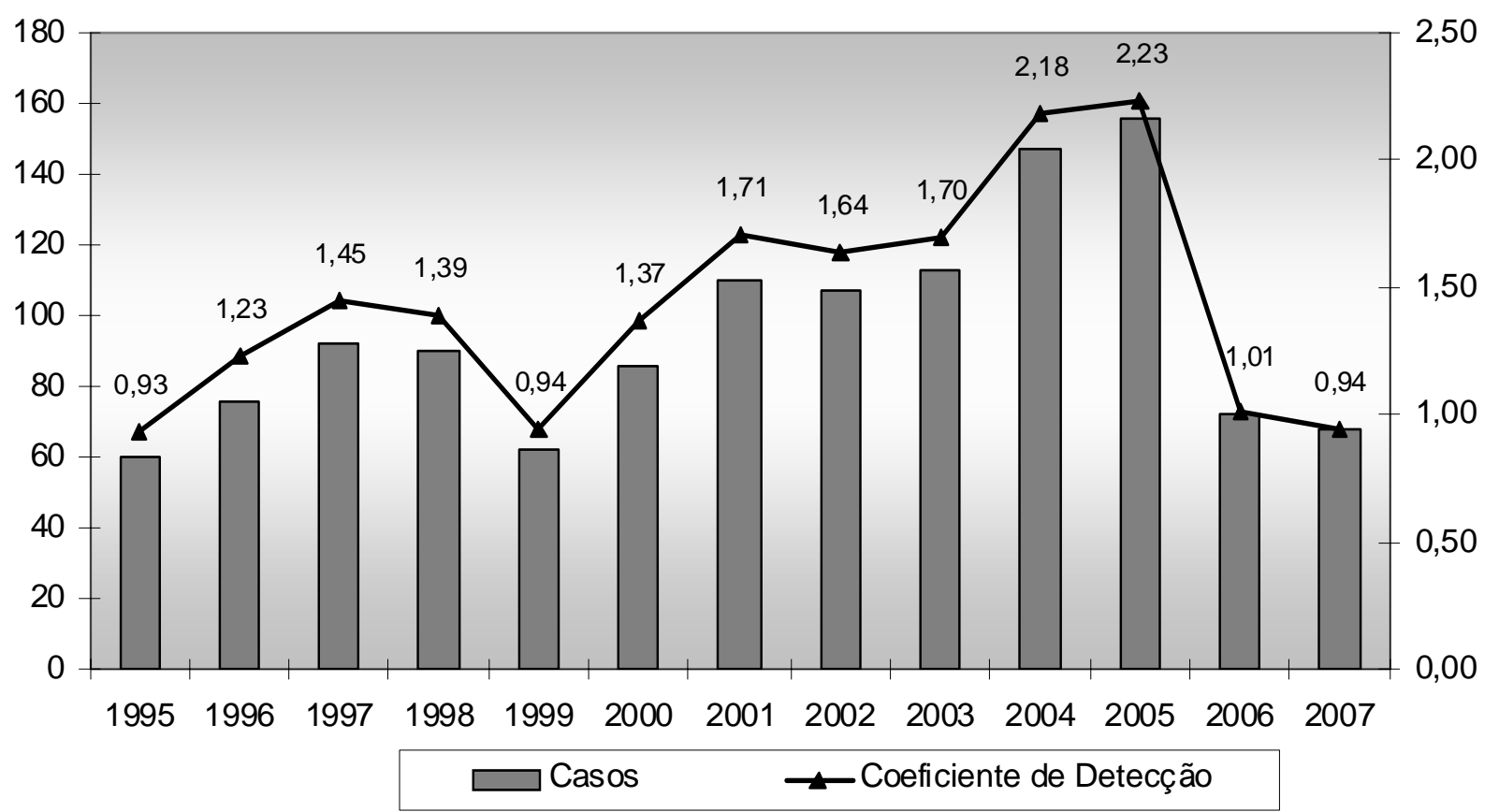

Fonte: SINANNET - SMS/Fortaleza (Dados Preliminares até 30/07/2008)

Gráfico 2. Casos novos e coeficiente de detecção de hanseníase na faixa etária menor de 15 anos no município de Fortaleza nos anos de 1995 : 
Hanseníase no município de Fortaleza, CE, Brasil: aspectos epidemiológicos e operacionais em menores de 15 anos no período de 1995 a 2006

por sexo com detecção de casos sofrendo alternância ao longo dos anos. A forma clínica tuberculóide é a mais freeüente, chegando a mais de $50 \%$ dos casos detectados no ano de 2006, e se mantendo em valores próximos a este nos demais anos. No ano de 2007 houve uma Queda neste percentual, mas deve ser destacada a elevada proporção de casos classificados como ignorados ou em branco (Tabela 1). A forma tuberculóide é característica em pessoas Que apresentam resistência ao $M$. leprae, mas Que sofreram contatos sucessivos e acabam por desenvolver a doença ${ }^{(17,18)}$. Assim a presença de uma elevada proporção nesta faixa etária indica uma necessidade de busca e exame de contatos.

Quanto à classe operacional, a forma paucibacilar é a mais freeüente em todos os anos, chegando a $67 \%$ das formas identificadas no ano de 2006. Apenas no ano de 2003 e 2007 houve uma equivalência nesta

Tabela 1. Distribuição dos casos de hanseníase em menores de 15 anos segundo sexo, forma clínica, classe operacional na notificação e modo de deteç̧ão no município de Fortaleza nos anos de 2001 a 2007.

\begin{tabular}{|c|c|c|c|c|c|c|c|c|c|c|c|c|c|c|c|}
\hline \multirow{3}{*}{$\begin{array}{ll} & \text { VARIÁVEL } \\
& \text { SEXO } \\
\end{array}$} & \multicolumn{14}{|c|}{ ANOS } & \multirow{3}{*}{ Total } \\
\hline & \multicolumn{2}{|c|}{2001} & \multicolumn{2}{|c|}{2002} & \multicolumn{2}{|c|}{2003} & \multicolumn{2}{|c|}{2004} & \multicolumn{2}{|c|}{2005} & \multicolumn{2}{|c|}{2006} & \multicolumn{2}{|c|}{2007} & \\
\hline & $\mathrm{N}$ & $\%$ & $\mathrm{~N}$ & $\%$ & $\mathrm{~N}$ & $\%$ & $\mathrm{~N}$ & $\%$ & $\mathrm{~N}$ & $\%$ & $\mathrm{~N}$ & $\%$ & $\mathrm{~N}$ & $\%$ & \\
\hline Masculino & 32 & 57,1 & 21 & 38,8 & 25 & 52,0 & 41 & 50,0 & 47 & 59,4 & 25 & 39,0 & 40 & 58,8 & 231 \\
\hline Feminino & 24 & 42,8 & 33 & 61,1 & 23 & 47,9 & 41 & 50,0 & 32 & 40,5 & 39 & 60,9 & 28 & 41,1 & 220 \\
\hline \multicolumn{16}{|l|}{$\begin{array}{l}\text { FORMA CLINICA } \\
\text { NA NOTIFICAÇÃO }\end{array}$} \\
\hline Ign/Branco & 0 & 0,0 & 0 & 0,0 & 5 & 8,9 & 2 & 3,5 & 0 & 0,0 & 0 & 0,00 & 8 & 14,2 & 15 \\
\hline Indeterminada & 5 & 8,9 & 8 & 14,8 & 0 & 0,0 & 10 & 12,2 & 7 & 8,8 & 5 & 7,8 & 8 & 11,7 & 43 \\
\hline Tuberculóide & 28 & 50,0 & 24 & 44,4 & 19 & 39,5 & 37 & 45,1 & 40 & 50,6 & 38 & 59,3 & 18 & 26,4 & 204 \\
\hline Dimorfa & 16 & 28,5 & 15 & 27,7 & 16 & 33,3 & 28 & 34,1 & 25 & 31,6 & 18 & 28,1 & 24 & 35,2 & 142 \\
\hline Virchowiana & 7 & 12,5 & 7 & 12,9 & 7 & 14,5 & 5 & 6,1 & 7 & 8,8 & 3 & 4,6 & 10 & 14,7 & 46 \\
\hline Não classificada & 0 & 0,0 & 0 & 0,0 & 1 & 2,0 & 0 & 0,0 & 0 & 0,0 & 0 & 0,0 & 0 & 0,00 & 1 \\
\hline \multicolumn{16}{|l|}{$\begin{array}{l}\text { CLASSE OPERACIONAL } \\
\text { NA NOTIFICAÇÃO }\end{array}$} \\
\hline Ign/Branco & 1 & 1,79 & 0 & 0,0 & 0 & 0,0 & 1 & 1,2 & 0 & 0,0 & 0 & 0,0 & 0 & 0,0 & 2 \\
\hline Paucibacilar & 31 & 55,3 & 32 & 59,2 & 23 & 47,9 & 50 & 60,9 & 48 & 60,7 & 43 & 67,1 & 32 & 47,0 & 259 \\
\hline Multibacilar & 24 & 42,8 & 22 & 40,7 & 25 & 52,0 & 31 & 37,8 & 31 & 39,2 & 21 & 32,8 & 36 & 52,9 & 190 \\
\hline \multicolumn{16}{|l|}{$\begin{array}{l}\text { MODO DE } \\
\text { DETECÇÃO }\end{array}$} \\
\hline Ign/Branco & 0 & 0,0 & 1 & 1,8 & 0 & 0,0 & 0 & 0,0 & 0 & 0,00 & 0 & 0,0 & 0 & 0,0 & 1 \\
\hline Encaminhamento & 37 & 66,0 & 47 & 87,0 & 36 & 75,0 & 59 & 71,9 & 61 & 77,2 & 51 & 79,6 & 50 & 73,5 & 341 \\
\hline Demanda espontânea & 13 & 23,2 & 3 & 5,5 & 7 & 14,5 & 16 & 19,5 & 8 & 10,1 & 5 & 7,8 & 10 & 14,7 & 62 \\
\hline Exame coletividade & 0 & 0,0 & 1 & 1,8 & 1 & 2,08 & 0 & 0,0 & 3 & 3,8 & 1 & 1,5 & 0 & 0,0 & 6 \\
\hline Exame contatos & 6 & 10,7 & 2 & 3,7 & 4 & 8,33 & 6 & 7,3 & 7 & 8,8 & 7 & 10,9 & 8 & 11,7 & 40 \\
\hline Outros modos & 0 & 0,0 & 0 & 0,0 & 0 & 0,00 & 1 & 1,2 & 0 & 0,0 & 0 & 0,0 & 0 & 0,0 & 1 \\
\hline \multicolumn{16}{|l|}{$\begin{array}{l}\text { UNIDADE DE SAÚDE } \\
\text { NOTIFICADORA }\end{array}$} \\
\hline Centro de Referência & 45 & 80,3 & 48 & 88,8 & 38 & 79,1 & 62 & 75,6 & 63 & 79,7 & 53 & 82,8 & 51 & 75,0 & 360 \\
\hline Hospital Universitário & 4 & 7,1 & 2 & 3,7 & 4 & 8,3 & 12 & 14,6 & 7 & 8,86 & 7 & 10,9 & 2 & 2,9 & 38 \\
\hline UBASF & 7 & 12,5 & 4 & 7,4 & 6 & 12,5 & 8 & 9,76 & 9 & 11,3 & 4 & 6,2 & 15 & 22,0 & 53 \\
\hline TOTAL & 56 & 100,0 & 54 & 100,0 & 48 & 100,0 & 82 & 100,0 & 79 & 100,0 & 64 & 100,0 & 68 & 100,0 & 451 \\
\hline
\end{tabular}

Tabela 2. Proporção de contatos examinados e registrados em menores de 15 anos no município de Fortaleza nos anos de 2002 a 2007.

\begin{tabular}{cccc}
\hline ANO & Contatos Registrados & Contatos Examinados & Proporção de Examinados/registrados \\
\hline 2002 & 0 & 10 & - \\
2003 & 67 & 15 & 22,4 \\
2004 & 175 & 85 & 48,6 \\
2005 & 264 & 93 & 35,2 \\
2006 & 245 & 107 & 43,7 \\
2007 & 225 & 134 & 59,6 \\
\hline TOTAL & $\mathbf{9 7 6}$ & $\mathbf{4 4 4}$ & $\mathbf{4 5 , 5}$ \\
\hline
\end{tabular}

Fonte: SINANNET - SMS/Fortaleza (Dados Preliminares até 30/07/2008) 
Tabela 3. Grau de incapacidade no diagnóstico e na cura dos casos novos de hanseníase detectados em menores de 15 anos de 2001 a 2007 - Fortaleza.

\begin{tabular}{|c|c|c|c|c|c|c|c|c|c|c|}
\hline \multirow[b]{2}{*}{ ANO } & \multirow{2}{*}{$\begin{array}{c}\text { Grau de } \\
\text { Incapacidade no } \\
\text { diagnóstico }\end{array}$} & \multicolumn{9}{|c|}{ Grau de Incapacidade na alta } \\
\hline & & NA & $\%$ & 0 & $\%$ & I & $\%$ & II & $\%$ & TOTAL \\
\hline \multirow{4}{*}{2001} & 0 & 6 & 13,6 & 24 & 54,5 & I & 2,2 & 0 & 0,0 & 44 \\
\hline & I & 0 & 0,0 & 0 & 0,0 & 1 & 100,0 & 0 & 0,0 & 1 \\
\hline & II & 0 & 0,0 & 2 & 33,3 & 1 & 16,6 & 2 & 33,3 & 6 \\
\hline & Não avaliado & 3 & 60,0 & 2 & 40,0 & 0 & 0,00 & 0 & 0,0 & 5 \\
\hline \multirow{4}{*}{2002} & 0 & 4 & 8,1 & 32 & 65,3 & 0 & 0,0 & 0 & 0,0 & 49 \\
\hline & I & 0 & 0,0 & 0 & 0,0 & 1 & 50,0 & 0 & 0,0 & 2 \\
\hline & II & 0 & 0,0 & 0 & 0,0 & 0 & 0,0 & 1 & 100,0 & 1 \\
\hline & Não avaliado & 2 & 100,0 & 0 & 0,0 & 0 & 0,0 & 0 & 0,0 & 2 \\
\hline \multirow{4}{*}{2003} & 0 & 7 & 16,6 & 28 & 66,6 & 0 & 0,0 & 0 & 0,0 & 42 \\
\hline & I & 0 & 0,00 & 0 & 0,0 & 1 & 50,0 & 0 & 0,0 & 2 \\
\hline & II & 1 & 50,0 & 1 & 50,0 & 0 & 0,0 & 0 & 0,0 & 2 \\
\hline & Não avaliado & 1 & 50,0 & 0 & 0,0 & 0 & 0,0 & 0 & 0,0 & 2 \\
\hline \multirow{4}{*}{2004} & 0 & 6 & 9,2 & 48 & 73,8 & 0 & 0,0 & 0 & 0,0 & 65 \\
\hline & I & 2 & 25,0 & 2 & 25,0 & I & 12,5 & 0 & 0,0 & 8 \\
\hline & II & 0 & 0,0 & 0 & 0,0 & 0 & 0,0 & 2 & 50,0 & 4 \\
\hline & Não avaliado & 2 & 40,0 & 1 & 20,0 & 0 & 0,0 & 0 & 0,0 & 5 \\
\hline \multirow{4}{*}{2005} & 0 & 14 & 19,7 & 49 & 69,0 & 1 & 1,4 & 0 & 0,0 & 71 \\
\hline & I & 0 & 0,0 & 1 & 33,3 & 0 & 0,0 & 0 & 0,0 & 3 \\
\hline & II & 1 & 50,0 & 0 & 0,0 & 0 & 0,0 & I & 50,0 & 2 \\
\hline & Não avaliado & 1 & 33,3 & 0 & 0,0 & 0 & 0,0 & 0 & 0,0 & 3 \\
\hline \multirow{4}{*}{2006} & 0 & 11 & 19,3 & 41 & 71,9 & 1 & 1,7 & 0 & 0,0 & 57 \\
\hline & I & 0 & 0,0 & 0 & 0,0 & 0 & 0,0 & 0 & 0,0 & 0 \\
\hline & II & 1 & 25,0 & 1 & 25,0 & 0 & 0,0 & 0 & 0,0 & 4 \\
\hline & Não avaliado & 3 & 100,0 & 0 & 0,0 & 0 & 0,0 & 0 & 0,0 & 3 \\
\hline \multirow{4}{*}{2007} & 0 & 31 & 59,6 & 17 & 32,6 & 2 & 3,8 & I & 1,9 & 52 \\
\hline & I & 4 & 66,6 & 1 & 16,6 & 1 & 16,6 & 0 & 0,0 & 6 \\
\hline & II & 2 & 66,6 & 1 & 33,3 & 0 & 0,0 & 0 & 0,0 & 3 \\
\hline & Não avaliado & 4 & 100,0 & 0 & 0,0 & 0 & 0,0 & 0 & 0,0 & 4 \\
\hline & Não avaliado & & \multicolumn{2}{|c|}{ Melhoraram o grau } & & & m o grau & & \multicolumn{2}{|c|}{ Aumentaram o grau } \\
\hline
\end{tabular}

proporção, com uma leve superioridade no número de casos da forma multibacilar (Tabela I). Esse cenário reflete a potencialidade de doença com maior gravidade nos indivíduos acometidos.

O modo de detecção encontrado em aproximadamente 75\% dos casos foi por meio de encaminhamento, isto se deve principalmente pela dificuldade no diagnóstico de hanseníase nesta faixa etária e pela ausência de uma rede de atenção primária capacitada e atuante no tocante ao diagnóstico desta enfermidade. Além disso, os dados apresentados revelam baixa Qualidade da abordagem de contatos das crianças, uma ação de grande relevância. Tal fato pode ser ainda mais sustentado pela proporção de casos diagnosticados e notificados pela unidade de saúde de referência para a hanseníase no município, Que abrange Quase $80 \%$ das notificações no período em Questão.

Ressalta-se Que nos casos diagnosticados nessa faixa etária, o contexto familiar é importante, devendo-se levar em consideração critérios mais rigorosos no estabelecimento de percentuais mínimos esperados de exames de contatos intradomiciliares ${ }^{(3)}$. Assim a proporção de contatos examinados dentre aqueles registrados deve ser aferida Tabela 2).

Segundo os dados do Ministério da Saúde a partir do "Exercício de Monitoramento da Hanseníase" (Leprosy Elimination Monitoring - LEM), uma análise externa realizada em 2005 em 16 capitais brasileiras, os dados de tendência dos coeficientes de prevalência em Fortaleza para o período de 2000 a 2004 mostram um comportamento instável e em níveis elevados ${ }^{(19)}$. Quando foi aplicado o critério da OMS (remoção da prevalência dos pacientes Que não compareceram ao serviço para receber tratamento por mais de 12 meses) observa-se uma Queda mais acentuada em todo o período. A razão prevalência/detecção em 2004 foi de 1,80, demonstrando Que há uma disparidade entre o número de casos diagnosticados Que entram no sistema e os Que saem por cura.

Ainda segundo o LEM, o município vem apresentando taxas de abandono ascendentes e elevadas. Este abandono explica, em parte, a manutenção da elevada prevalência. Em 2004 a taxa de abandono foi de $34,9 \%$. A proporção de pacientes em abandono do tratamento de hanseníase em Fortaleza de 2000 a 2004, segundo os dados do LEM, observou-se uma variação de $9,6 \%$ em 2000 a 34,9\% em 2004 (200l - 26,0\%, 2002 - 24,6\%, 2003-28,6\%)

Segundo esses mesmos dados, o coeficiente de detecção geral para o período de 2003 a 2004 mantém-se em nível de endemicidade 
muito alto (respectivamente, 3,98 e 3,67 por 10.000 habitantes). lá o coeficiente de detecção em menores de 15 anos para o mesmo período, apresentou um aumento de um ano para outro e mantémse com coeficiente muito alto, demonstrando transmissão ativa. Nos realizado em sua grande maioria pelo centro de referência do estado; as unidades básicas de saúde não apresentam desempenho satisfatório para a detecção de casos em menores de 15 anos. Esse cenário pode acarretar maior menor número de contatos examinados, retardo diagnóstico, possibilidade de incapacidades ao diagnóstico e ao final do tratamento polieuimioterápico, ampliando a dimensão do problema em termos individuais e coletivos.

Considerando-se a população deste estudo, a incidência de todos esses eventos certamente terá implicação durante toda a sua vida, tanto em termos físicos, Quanto psicológicos, sociais e comportamentais.

dados coletados pelo LEM as crianças representaram 7,9\% dos casos novos de 2004, semelhantes aos dados da coordenação municipal $(7,7 \%)$.

Deve ser destacada a elevada proporção de casos não avaliados tanto no momento da notificação e na alta, estes valores poderão mascarar a realidade Quanto ao grau de incapacidade nos menores de 15 anos, como demonstrado na Tabela 3. Isso compromete sobremaneira a avaliação do desenvolvimento das ações do programa de controle.

Isto se dá uma vez Que a prevenção de incapacidades é uma atividade fundamental desde o diagnóstico, durante o tratamento e em alguns casos, após a alta do tratamento polieuimioterápico. Por isso, é importante Que a pessoa com hanseníase seja orientada para a prática diária do auto-cuidado e a realização dos exercícios de prevenção e reabilitação, Quando necessários ${ }^{(3)}$.

As incapacidades físicas possibilitam a presença de estigma em relação aos indivíduos portadores da hanseníase, além de diminuição na participação social, ampliação da vulnerabilidade social, limitações para atividades físicas e problemas psicológicos ${ }^{(20)}$. Observando a coorte dos casos menores de 15 anos em Fortaleza, verifica-se que de forma geral não houve aumento no grau de incapacidade dos indivíduos Quando avaliados na notificação e no momento da alta. A Tabela 3 mostra a evolução dos casos e a proporção de portadores Que tiveram redução no grau de incapacidade, mantiveram o mesmo grau ou Que aumentaram a incapacidade física após o tratamento. Entretanto, o número de casos não avaliados compromete o desenvolvimento de atenção integral à saúde para essa população.

\section{CONCLUSÕES}

O presente estudo ressalta a importância do coeficiente de detecção em menores de 15 anos, considerada pelo Ministério da Saúde como um dos indicadores epidemiológicos estratégicos para análise do padrão da hanseníase em uma área específica.

Fortaleza mantém-se ao longo do período do estudo com coeficientes expressivos de detecção considerados para essa faixa etária. Operacionalmente, o alto percentual de casos diagnosticados com algum grau de incapacidade física e/ou com formas clínicas multibacilares revela a dinâmica de transmissão da doença e a manutenção da endemia. Este contexto possibilita um contato precoce de crianças ao $M$. leprae.

O principal modo de detecção se dá por encaminhamento, sendo realizado em sua grande maioria pelo centro de referência do estado; as unidades básicas de saúde não apresentam desempenho satisfatório para a detecção de casos em menores de 15 anos. Esse cenário pode acarretar maior menor número de contatos examinados, retardo diagnóstico, possibilidade de incapacidades ao diagnóstico e ao final do tratamento poliquimioterápico, ampliando a dimensão do problema em termos individuais e coletivos.

Considerando-se a população deste estudo, a incidência de todos esses eventos certamente terá implicação durante toda a sua vida, tanto em termos físicos, Quanto psicológicos, sociais e comportamentais.

\section{REFERÊNCIAS}

1. World Health Organization. Global leprosy situation, beginning of 2008. Weekly Epidemiol Record 2008; 83(33): 293-300.

2. World Health Organization. Global Strategy for further reducing the leprosy burden and sustaining leprosy control activities: plan period: 2006-2010. Geneva: WHO; 2005.

3. Ministério da Saúde (BR). Departamento de Atenção Básica Secretaria de Atenção à Saúde. Cad Atenção Básica 2007; 21 .

4. Ministério da Saúde (BR). Secretaria de Vigilância em Saúde. Programa Nacional de Controle da Hanseníase. Relatório Executivo do PNCH. - Período Maio de 2007 a Junho de 2008 Brasília: Ministério da Saúde; 2008.

5. Martelli CMT, Stefani MMA, Penna G, Andrade ALSS. Endemias e epidemias brasileiras, desafios e perspectivas de investigação científica: hanseníase. Rev Bras Epidemiol 2002; 5(3): 273-85.

6. Matos HID. Epidemiologia da hanseníase em coorte de contatos intradomiciliares no Rio de Janeiro (1987-1991). Cad Saúde Pública 1999; 15(3): 533-42.

7. Albuquerque MFPM, Morais HMM, Ximenes R. A expansão da hanseníase no nordeste brasileiro. Rev Saúde Pública 1989; 23(2): 107-16.
8. Montenegro AC, Werneck GL, Kerr-Pontes LR, Barreto ML, Feldmeier $\mathrm{H}$. Spatial analysis of the distribution of leprosy in the State of Ceara, Northeast Brazil. Mem Inst Oswaldo Cruz 2004; 99(7): 683-6.

9. Kerr-Pontes L R, Montenegro A C, Barreto M L, Werneck G L, Feldmeier $\mathrm{H}$. Inequality and leprosy in Northeast Brazil: an ecological study. Int I Epidemiol 2004; 33(2): 262-9.

10. Kerr-Pontes L R, Barreto M L, Evangelista C M, Rodrigues L C, Heukelbach J, Feldmeier H. Socioeconomic, environmental, and behavioural risk factors for leprosy in North-east Brazil: results of a case-control study. Int I Epidemiol 2006; 35(4): 994- 1000.

1 I. Ramos IR AN, Heukelbach J, Gomide M, Hinders DC, Schreuder PA. Health systems research training as a tool for more effective Hansen's disease control programmes in Brazil. Lepr Rev 2006; 77(3): 175-88.

12. Bakirtzief $Z$. Identificando barreiras para aderência ao tratamento de hanseníase. Cad Saúde Pública 1996; 12(4): 497-505.

13. Moraes MO, Cardoso CC, Vanderborght PR, Pacheco AG. Genetics of host response in leprosy. Lepr Rev 2006; 77(3): 189-202. 
Alencar CHM, et al.

14. Lockwood D N. Suneetha S. Leprosy: too complex a disease for a simple elimination paradigm. Bull World Health Organ 2005; 83(3): 230-5.

15. Façanha MC, Pinheiro AC, Lima JRC, Ferreira MLLT, Teixeira GFD, RouQuayrol MZ. Hanseníase: subnotificação de casos em Fortaleza - Ceará, Brasil. An Bras Dermatol 2006; 81 (4): 329-33.

16. Gallo M E N, Ramos IR A N, Albueuerque E C A, Nery I A C, Sales A M. Alocação do paciente hanseniano na poliQuimioterapia: correlação da classificação baseada no número de lesões cutâneas com os exames baciloscópicos. An Bras Dermatol 2003; 78(4): 415-24.
17. Lana FC, Amaral EP, Lanza FM, Lima PL, de Carvalho AC, Diniz LG. Hanseníase em menores de 15 anos no Vale do JeQuitinhonha, Minas Gerais, Brasil. Rev Bras Enferm 2007; 60(6): 696-700.

18. Ferreira I N, Alvarez RRA. Hanseníase em menores de quinze anos no município de Paracatu, MG (1994 a 200 I). Rev Bras Epidemiol 2005;8(1):41-49.

19. Pan American Health Organization. Situation Report: Leprosy in the Americas, 2007. Disponível em: http://amro who int/ English/AD/DPC/CD/lep-sit-reg-2007pdf 2007/

20. Aquino DMC, Caldas AJM, Silva A A M, Costa JML. Perfil dos pacientes com hanseníase em área hiperendemica da Amazônia do Maranhão, Brasil. Rev Soc Bras Med Trop 2003; 36(1): 57-64. 